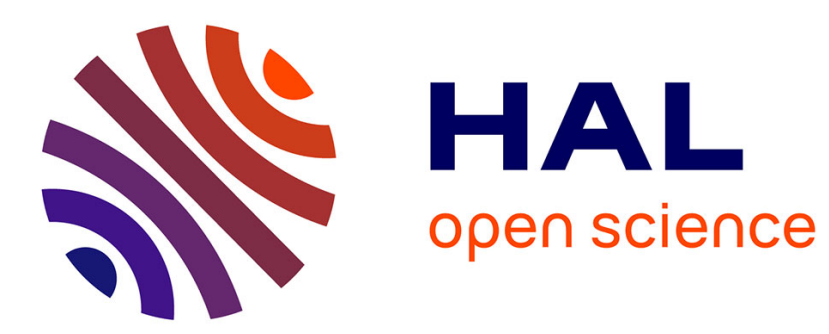

\title{
La réforme des entreprises d'État chinoises, une entrée par la comptabilité : 1993-2001
}

Marc Amblard, Corinne Eyraud

\section{To cite this version:}

Marc Amblard, Corinne Eyraud. La réforme des entreprises d'État chinoises, une entrée par la comptabilité : 1993-2001. Comptabilité - Contrôle - Audit, 2008, pp.27-42. halshs-00377468

\section{HAL Id: halshs-00377468 \\ https://shs.hal.science/halshs-00377468}

Submitted on 23 Apr 2009

HAL is a multi-disciplinary open access archive for the deposit and dissemination of scientific research documents, whether they are published or not. The documents may come from teaching and research institutions in France or abroad, or from public or private research centers.
L'archive ouverte pluridisciplinaire HAL, est destinée au dépôt et à la diffusion de documents scientifiques de niveau recherche, publiés ou non, émanant des établissements d'enseignement et de recherche français ou étrangers, des laboratoires publics ou privés. 


\section{La réforme des entreprises d'État chinoises,}

une entrée par la

comptabilité : 1993-2001

The reform of State's Chinese enterprises, an accouting angle: 1993-2001

Marc AMBLARD* et Corine EYRAUD**

\section{Résumé}

La fonction première de la comptabilité est d'enregistrer et de communiquer un ensemble d'informations financières relatives à un objet prédéterminé, le plus souvent une entité économique. Qu'elles résultent de négociations collectives ou de prescriptions autoritaires, les règles comptables présentent la particularité d'évoluer pour laisser place à de nouveaux modèles de représentation. Si ces évolutions sont la conséquence de changements qui lui sont extérieurs, elles ne sont pas elles-mêmes sans effet sur les structures économiques, sociales et sociétales.

\section{Abstract}

Recording and communicating information about an economic entity are the primary function of accounting. The accounting rules result from collective negociations or autoritarian dictates; so, both present particular features: they evolve and give way to new models. We think these evolutions are the consequence of outside change; conversely, we also think they have an effect on economic, social and societal structures. The study of chinese accounting reform, few years ago, is an illuminating illustration.

* Maître de conférences HDR, membre du GREDEG UMR-6227 - Chercheur associé à L'IREBS-EBS Paris

** Maître de conférences, Université de Provence 
L'étude de la réforme comptable chinoise, il y quelques années, nous offre à cet effet une illustration éclairante.

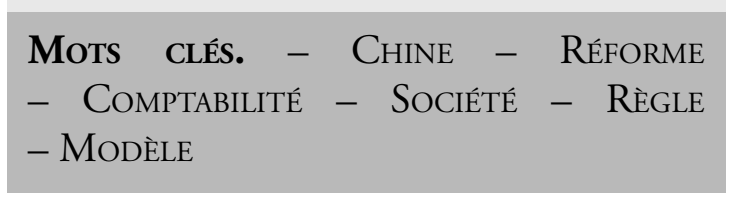

Correspondance :

\section{Marc Amblard \\ GREDEG UMR-6227 \\ CNRS-UNSA}

Université de Nice Sophia Antipolis

250, rue Albert Einstein

Bâtiment 2

06560 Valbonne

marcamblard@orange.fr
KEYWORDS. - CHINE - REFORM

ACCOUNTING - RULE - MODEL

\section{Introduction}

L'idée que la comptabilité est un construit historique et social nous semble aujourd'hui reconnue par une large partie de la science comptable même si finalement elle n’a commencé à faire son chemin que récemment. Comme l'a souligné M. Capron (2005, p. 6), « sous les apparences d'une technique - plus ou moins complexe ou ésotérique aux yeux des profanes -, la comptabilité est en fait un ensemble de constructions sociales, historiquement datées et génératrices d'effets économiques. L'idée d'une neutralité des instruments comptables a fait long feu... ".

Cette posture a d'ailleurs donné corps depuis une trentaine d'années à un programme de recherche en Grande-Bretagne autour de la revue Accounting, Organizations and Society ${ }^{l}$ et, en grande partie, dans le cadre de la London School of Economics ${ }^{2}$. Dans le même temps, de nombreux travaux français développaient cette conception socialisée de la comptabilité, même s'ils s'inscrivaient moins nettement dans une « école » unifiée ${ }^{3}$.

Nonobstant ces trois décennies de réflexion, A. Hopwood (2000) déplorait que les aspects institutionnels et sociaux de la comptabilité restent encore relativement inexplorés et notre savoir en la matière plutôt modeste. Ce programme scientifique rencontre effectivement différents obstacles ; en particulier parce que sa mise en ouvre nécessiterait des échanges entre la recherche comptable et les sciences sociales, ainsi qu'une immersion effective et longue sur le terrain étudié. Or, la comptabilité en est historiquement et institutionnellement très éloignée, beaucoup plus qu'elle ne l'était de la finance ou de la science économique avec qui les rapprochements, bien que fructueux, ne se sont pourtant pas faits sans résistance. Les sciences sociales, pour leur part, une fois achevés les travaux pionniers de Marx, Sombart et Weber, se sont très peu intéressées à la question comptable (Miller 2000). Ceci, nous semble-t-il, pour deux raisons. La première est liée aux compétences techniques 
nécessaires dont peu de spécialistes en sciences sociales étaient pourvus. La deuxième tient à l'acceptation par ces disciplines de la séparation entre ce qui est de l'ordre du social et ce qui est de l'ordre du technique ou de l'économique (Eyraud 2004).

Nous nous inscrivons ici dans le cadre d'une conception historique et socialisée de la comptabilité. Notre contribution porte sur trois points. Tout d'abord les spécificités de notre terrain et la période pendant laquelle il a été étudié le rendent particulièrement intéressant : "l'école anglaise " a souligné l'intérêt heuristique d'analyser les moments de changements comptables importants ${ }^{4}$ pour montrer l'historicité de la comptabilité ainsi que la matrice sociale et institutionnelle dans laquelle elle s'insère.

Ensuite, nous situons l'analyse à un moment charnière : non seulement il s'agit d'une réforme comptable majeure, mais également d'un passage d'une économie planifiée à un début d'économie de marché qui correspond à une profonde réorganisation de la société dans son ensemble. "Accounting innovation is to be understood by analyzing the complex interplay between the multiple arenas within which new ways of accounting emerge " (Miller 1994, p. 27). La plupart du temps, ce sont les dimensions économiques ou intra-organisationnelles qui sont mises en évidence. Ici, nous avons la chance de pouvoir en analyser les dimensions et les fondements sociaux voire sociétaux. Nous insisterons largement sur cet aspect.

Enfin, nous sommes également en mesure d'aborder les effets d'un changement comptable sur les représentations et les pratiques des acteurs, du fait des types de méthodes utilisées mais aussi de la temporalité de l'enquête (presque 10 ans) et la possibilité qui nous fut offerte de rencontrer certains acteurs plusieurs années de suite.

L'article, précisons-le, s'appuie sur six enquêtes se déroulant entre 1993 et $2001^{5}$. Trois méthodes de collecte, parfois concomitantes, ont été utilisées :

- un recueil documentaire;

- la réalisation d'une centaine d'entretiens ;

- l'observation participante à deux colloques internationaux.

C'est sur le caractère socialement construit du modèle comptable et sur son historicité que nous voudrions ici mettre l'accent. À cette fin, la première partie de l'article fournira une description du système comptable chinois cher à l'économie planifiée (1) ; sa réforme mise en œuvre depuis 1993 fera ensuite l'objet d'une lecture sociologique (2). Il s'agit de deux modèles et de deux systèmes qui reposent sur des règles différentes; ils peuvent ainsi nous servir de révélateurs.

\section{1. Économie planifiée et modèle comptable ${ }^{6}$}

L'histoire de la comptabilité chinoise ne commence pas avec l'économie planifiée ; la tenue des livres de comptes fut effective dès que les marchands eurent accès à une culture écrite (Goody 1999). Son rôle fut aussi amplement renforcé par l'État « dont les buts sont d'assurer une bonne utilisation des fonds publics par l'entreprise et une source stable de rentrées fiscales (Ding 2001, p. 36) ». Mais c'est à partir du $\mathrm{XX}^{\mathrm{e}}$ siècle, que se développe une comptabilité complexe utilisant progressivement de nombreux aspects et principes de la partie double, jusqu'à son adoption officielle et sa diffusion pendant la période républicaine (1911-1949). Les années suivantes sont alors le théâtre de nombreux

Comptabilité - Contrôle - Audit / Tome 14 - Volume 2 - Décembre 2008 (p. 27 à 42) 
changements qui vont profondément marquer la société chinoise : mise en place du premier plan quinquennal (1953) fondé sur une logique d'économie planifiée et transformation des entreprises privées en entreprises d'État. La création d'une vaste administration économique et statistique, largement inspirée à ses débuts du modèle soviétique (Eyraud 1999, p. 60-61) accompagne le processus. C'est dans ce contexte que seront élaborés de nouveaux cadres comptables.

\subsection{Entité et revenu}

Pendant la période de l'économie planifiée, les entreprises d'État chinoises émargent au budget de l'État; celui-ci finance les besoins de trésorerie et les investissements, absorbe les profits et éponge les pertes. Les entreprises ne sont finalement qu'un simple sous-ensemble de l'économie nationale et du budget national. Leur système comptable qui relève du domaine de la comptabilité publique ${ }^{7}$ repose sur la notion de fonds ; document essentiel, la "balance de fonds ", se compose de deux parties équivalentes (fonds alloués et fonds utilisés), chacune divisée en trois catégories : fonds fixes, fonds circulants et fonds spéciaux. La gestion financière des entreprises doit obéir au principe « d'utilisation des crédits conformément à leur affectation ».

Les définitions de nombreuses catégories comptables sont liées aux concepts de l'économie politique marxiste, tels qu'élaborés par l'URSS, à partir de la pensée de Marx, dans ses débuts d'économie planifiée. Les fonds fixes, par exemple, sont définis comme « les fonds utilisés par l'entreprise en tant que moyens de travail »; ils comprennent les bâtiments professionnels, les équipements, les machines mais aussi tout " ce qui rend possible pour les travailleurs la réalisation de leur travail sur une longue période " : logements, crèches et écoles pour les enfants, dispensaire, etc. Les limites de l'entité économique sont ici repoussées pour y intégrer des actifs qui ne concourent pas directement à la création de richesse mais y sont liés du fait qu'ils participent indirectement au maintien et au développement de la force de travail. Le phénomène peut surprendre mais il n'est pas nouveau, les frontières de l'objet comptable sont purement conventionnelles, elles varient au gré des choix politiques, économiques et idéologiques qui sous-tendent le modèle (Amblard 2000). Il s'agit là d'une autre idée et d'une autre forme d'entreprise que celle que nous connaissons aujourd'hui en Occident et que découvrent depuis peu les chinois.

Si la perception comptable de la firme dans son étendue est surprenante, la mesure du revenu ne l'est pas moins. L'entreprise assume une part des dépenses privées réalisées par son personnel (hébergement, nourriture...) ; aussi, ces charges, en tant que liées à l'entretien de la force de travail, sont intégrées aux coûts de production. Dans la théorie marxiste, le profit est la valeur créée par les travailleurs. Les impôts sont donc considérés comme une part du profit transféré à l'État, les frais financiers comme une part transférée aux banques. Dans le système comptable de l'économie planifiée et ce jusqu’à la réforme de 1993, le bénéfice ou profit comprend ainsi :

- les impôts et taxes diverses ;

- les frais financiers (ils sont rares mais existent);

- le profit distribué aux employés par le fonds de bien-être (dédié à la construction de logements, crèches, écoles, etc.) et par les primes (mais pas les salaires) ;

- le profit remis à l'État. 
Ces catégories de dépenses ne sont donc pas considérées comme des charges mais comme des répartitions du bénéfice (Eyraud 1999, p. 268). Rappelons-le, si les frontières de l'entité économique sont conventionnelles, la mesure du profit l'est tout autant : les charges et les produits qui déterminent le résultat ne relèvent pas d'une vérité immanente mais résultent de choix à un moment donné (Amblard 2002). Loin d'être neutres, ces qualifications comptables privilégient un point de vue particulier. Là, il s’agit du Parti.

\subsection{Autorité macro-économique et contrôle micro-économique}

La comptabilité des entreprises s'intègre dans le système particulier qu'est celui de l'économie planifiée. Son objectif est macro-économique puisqu'elle doit fournir des informations pour la planification nationale ; il est aussi micro-économique attendu que la comptabilité permet de contrôler la réalisation du plan par l'entreprise et l'application de la discipline financière édictée par l'État et les autorités locales.

Compte tenu de l'objectif macro-économique, le lien entre système comptable et système statistique est très fort : ce sont, le plus souvent, les catégories statistiques qui informent les catégories comptables. Ainsi, le dispositif statistique d'État comprend un poste intitulé « montant global des marchandises au détail dans la société ". Pour obtenir cette donnée, il est demandé aux entreprises industrielles et commerciales de fournir dans leurs documents comptables outre leur chiffre d'affaires global (en valeur et en volume), un montant ne comprenant que les produits achetés par les clients pour être consommés directement par eux-mêmes dans leur propre cycle de production. Ce poste appelé " montants nets des ventes " (en traduction littérale) exclut donc les biens achetés à l'entreprise pour être revendu en l'état. "Le montant global des marchandises au détail dans la société » équivaut alors au total des «montants nets des ventes » de toutes les firmes du pays ; cette donnée est d'abord exploitée pour décider de l'augmentation ou de la diminution de telle production d'un point de vue national ou local, voire de transferts de tel produit entre provinces ou municipalités ; il est ensuite utilisé pour vérifier l'adéquation entre la destination prévue par le plan et la destination effective. Cette subordination du système comptable au système statistique se retrouve également dans la comptabilité bancaire : les documents comptables des banques sont ainsi élaborés secteur industriel par secteur industriel, dans un même souci d'information au service de la planification et de la vérification.

D’un point de vue micro-économique cette fois-ci, le contrôle sur la firme se réalise essentiellement par l'application du principe "d'utilisation des crédits conformément à leur affectation "; ce contrôle est aussi exercé par la banque avec laquelle l'entreprise chinoise entretient des relations financières sachant qu'elle doit obligatoirement ouvrir ses comptes dans le bureau de banque de son district. Elle détient un compte pour chaque type de fonds (fixes ou circulants) ainsi que pour les fonds spéciaux. Elle doit fournir à sa banque son plan d'achat, de vente, de coût, son plan financier ainsi que ses documents comptables mensuels, trimestriels et annuels. Sur le plan d'achat figurent les matériaux à acheter, leurs prix et l'identité des fournisseurs ; sur le plan de vente les produits à vendre, leurs prix et l'identité des clients; enfin, sur le plan financier, l'utilisation prévue de chaque fonds. Jusquau début des années 1990, il n'existe en Chine qu'un seul moyen de paiement ${ }^{8}$ : charger la banque, en lui fournissant la facture, d'aller se faire payer auprès de celle du client (ou d'aller payer celle du fournisseur). Il est donc facile pour l'établissement financier de vérifier que l'entreprise se conforme bien au plan. Finalement, la banque chinoise non seulement contrôle les entreprises mais assure également leur gestion financière.

Comptabilité - Contrôle - Audit / Tome 14 - Volume 2 - Décembre 2008 (p. 27 à 42) 


\subsection{Rôle et trajet de l'information comptable}

Il existe alors plus d'une cinquantaine de "systèmes comptables ». Chacun comprend un plan de compte, un modèle type de document ainsi que des textes très détaillés et directifs expliquant le contenu de chaque item, sa méthode d'enregistrement, etc. Ces systèmes qui ont valeur de règlement diffèrent selon l'entreprise (collective ou d'État) et selon le secteur de production puisque chaque ministère industriel les élabore à partir des directives du ministère des Finances et du Bureau National des Statistiques. La division comptabilité du ministère industriel (ministère de la Sidérurgie par exemple) les transmet aux services comptabilité des bureaux provinciaux de la sidérurgie qui eux-mêmes les transmettent à leur tour aux services comptabilité des bureaux municipaux de la sidérurgie ; ces derniers les communiquent enfin aux sections comptabilité des entreprises qui leur sont rattachées.

L'information comptable effectue le chemin inverse: les documents mensuels, trimestriels et annuels sont remis par l'entreprise à son autorité de tutelle (par exemple le bureau municipal de la sidérurgie), à sa banque et au bureau des Finances. Il est quasiment impossible pour tout autre organisme (entreprise ou administration) d'obtenir ces documents. Ceux-là permettent à l'autorité de tutelle d'évaluer le degré de réalisation du plan de toutes les entreprises qui lui sont subordonnées. Ladite autorité remet ensuite ces données agrégées au Bureau Local des Statistiques et au Bureau Provincial de la Sidérurgie. Enfin, l'information comptable remonte encore un échelon pour parvenir entre les mains du Bureau Provincial des Statistiques et au ministère de la Sidérurgie.

On retrouve dans ce mode de gestion de l'information et des données comptables et financières, les principaux éléments du système chinois de gestion «échelon par échelon » de l'économie et, plus largement, de la société (Eyraud 1999) dans son ensemble. Il faut comprendre qu'il existe très peu de lois, tout est encadré par des règlements transmis en suivant les lignes administratives qui relient hiérarchiquement les organisations chinoises; précisons qu'ils peuvent être modifiés (en raison de conditions sectorielles ou locales particulières) par un ministère pour son secteur industriel ou par les échelons provinciaux et municipaux. Il s'agit là d'un type de gestion très particulariste et non pas universaliste fondé sur un droit national. Les entreprises reçoivent ainsi tous les règlements qui encadrent leurs activités, par exemple tout ce qui concerne l'embauche, les salaires, les conditions de travail, d'hygiène, etc.

Ce mode de gestion, de surcroît très précis et très détaillé, réduit très largement les possibilités de choix au niveau de l'organisation (ici au niveau de l'entreprise et en matière d'enregistrement comptable).

Pour autant, aucune règle n'est immuable et les années 1990 vont être la scène de profondes évolutions tant dans la société dans son ensemble que dans les entreprises. Le modèle comptable n'échappera pas au mouvement. Dans une certaine mesure, il en sera même un des acteurs.

\section{2. Économie de marche et réforme comptable}

À partir du début des années 1980, le monde académique du pays commence à avoir accès aux théories et aux systèmes comptables occidentaux, en particulier anglo-saxons. Le projet de réformer le système chinois commence à animer les esprits du Parti et de certains responsables d'entreprises. 
Différents groupes d'experts et commissions sont alors constitués suivis de colloques internationaux organisés sur ce thème. C'est finalement en 1993 que de nouveaux règlements à l'appui de cette réforme comptable entrent en vigueur'. L'idée générale est explicite : il s'agit de «se connecter avec les pratiques internationales ». Des acteurs internationaux vont alors jouer un rôle important et favoriser cette connexion à l'instar de la Banque mondiale qui accordera un prêt à la Chine pour financer le projet. Une condition toutefois : le pays doit accepter une société d'expertise désignée par elle en tant que conseiller auprès du ministère des Finances; ce sera l'un des cinq grands cabinets anglo-saxons.

La Chine est alors engagée dans un processus de réforme majeure dont elle ne sortira pas en raison notamment des pressions qui s'exercent sur le nouveau modèle (Ding 2001) : pressions internes du fait de la volonté des autorités chinoises de réorienter la destinée du pays mais aussi externes par l'ouverture du pays aux entreprises étrangères qui s'y implantent et des firmes locales qui s'expatrient ou se financent sur les grandes places boursières mondiales.

\subsection{Changement d'objectifs et découplage comptabilité-statistique}

Ces nouveaux règlements ${ }^{10}$ introduisent tout d'abord un changement de portée générale : l'information comptable doit être élaborée dans le but de "répondre aux besoins du contrôle macro-économique, des utilisateurs extérieurs et de la gestion financière des entreprises". Les états financiers se transforment ainsi en documents publics, ouverts aux agents extérieurs et non plus aux seuls bureaux administratifs concernés. Pour leur part, les documents de comptabilité analytique deviennent des données internes que l'entreprise ne remet plus obligatoirement aux départements administratifs et que le directeur organise à sa convenance. La comptabilité change donc officiellement d'objectif et de destinataires : il ne s'agit plus seulement de fournir des informations pour la planification à venir et pour le contrôle de celle en cours, mais de présenter des données économiques sur l'entreprise. Celles-ci peuvent désormais être utilisées comme un outil de gestion par la direction et permettre une évaluation de l'entreprise par ses différents partenaires.

L'information comptable se détache alors de l'information statistique. Durant l'économie planifiée, les plans comptables et les modèles standard des documents étaient définis par les ministères industriels sous la direction du bureau national des Statistiques et du ministère des Finances. Dans les années 1980, ils sont élaborés dans une difficile négociation entre les différents acteurs, notamment le ministère industriel promouvant une comptabilité ancrée dans une logique économique occidentale et le bureau national des Statistiques attaché quant à lui à une comptabilité orientée vers la planification et les statistiques nationales. La logique entrepreneuriale finit par l'emporter : à partir de 1993, les documents comptables et statistiques sont dissociés et le bureau national des Statistiques n'intervient plus dans l'élaboration des systèmes comptables. Un de nos interlocuteurs, employé d'une banque, nous explique cette réforme : " avant 1992, nous subissions l'influence de la planification, les items de notre balance de fonds étaient tous divisés en fonction du secteur : chimie, textile, etc. Pour nous banquiers, cela n'avait aucune utilité contrairement au bureau national des Statistiques; nous ce qui nous intéresse c'est de savoir par exemple s'il s'agit de comptes à vue ou de comptes à échéance fixe ; à présent, nous possédons ces informations. "

À la fonction redditionnelle du modèle comptable, s'ajoute progressivement un rôle décisionnel.

Comptabilité - Contrôle - Audit / Tome 14 - Volume 2 - Décembre 2008 (p. 27 à 42) 
Marc AMblard et Corine Eyraud

\subsection{Système comptable réformé, entreprise repensée et nouveau mode de gestion du social}

Les règlements promulgués en 1993 définissent les nouveaux concepts utilisés en comptabilité : actif, passif, capitaux propres, produit, charge, profit et perte. Ils définissent également les nouveaux documents comptables largement inspirés du modèle anglo-saxon : on compte désormais un bilan, un compte de résultat et un tableau de financement. La séparation que le nouveau système instaure entre les comptabilités externe et analytique s'appuie sur une vision hétérodoxe de l'entreprise : une entité patrimoniale et non plus un simple élément du système économique national et du budget de l'État. La comptabilité chinoise devient ainsi une comptabilité d'entreprise au sens capitaliste du terme : les concepts de frontière et de revenu sont littéralement bouleversés ${ }^{11}$. C'est ainsi qu'à terme, les immobilisations non professionnelles (logements des employés, crèches, etc.) par exemple n'ont plus à figurer dans le bilan des entreprises. La firme ne doit plus supporter mais externaliser à la fois les coûts de production de la force de travail et la gestion de cette production. Aussi, de telles charges seront progressivement transférées aux collectivités publiques (municipalités, provinces, État). Concomitamment, ces dernières instaurent un système de retraite par capitalisation, une caisse d'assurance maladie (jusque là assumée directement par l'entreprise), une couverture contre les risques de chômage (les surplus de main d'œuvre étaient aussi gérés par l'entreprise) et enfin une individualisation des coûts de formation (école publique, obligatoire et payante).

De manière plus générale, on assiste à une réinvention du social par la mise en place d'une administration autonome et spécialisée qui supplante une fonction sociale certes existante mais jusqu'à présent encastrée dans l'entreprise d'État. Celle-ci n’aura donc plus à en supporter le coût. C'est l'esprit et la finalité mêmes de la firme chinoise qui subissent ici une profonde métamorphose.

Ainsi, là où il y avait auparavant un seul système économique national et où les différentes dimensions de l'activité de production étaient logées dans l'entreprise, on assiste à une "autonomisation " des fonctions. Des frontières se créent, des découpages, des discontinuités entre les entreprises ellesmêmes (chacune ayant son bilan et ses capitaux propres), mais aussi entre les entreprises et les départements administratifs, les banques d'État, les services sociaux... Ces séparations que Robert Salais (1986) a appelé internalités et externalités ${ }^{12}$ de l'entreprise sont à présent inscrites, objectivées dans les nouveaux documents, règles et classifications comptables. Ceux-ci sont au demeurant applicables à toutes les firmes installées sur le sol chinois, quels que soient leur statut et leur secteur d'activité. Les différents systèmes comptables devraient disparaître pour laisser place à un seul plan comptable applicable à tous. En cela, cette réforme s'inscrit dans un changement fondamental de mode de gestion de la société : le passage d'une micro-gestion particulariste de l'échelon inférieur par l'échelon supérieur à une gestion universaliste par la loi et par des organismes spécialisés dans une activité donnée (ici le ministère des Finances et les organismes professionnels comptables).

\subsection{L'impact social de la réforme comptable}

Les travaux des ethnométhodologues sur le rôle que jouent les textes et documents dans la construction de la réalité tout comme les travaux (Thévenot (1988), Desrosières, Salais et ali (1986), Boltanski (1982) entre autres) sur les "catégorisations sociales »" s'accordent à reconnaître que ces formes sociales objectivées, en ce qu'elles véhiculent des modèles de représentation de la réalité, ont un effet 
" en retour » sur la réalité sociale : elles coordonnent, structurent les activités et orientent les anticipations des acteurs. De la même façon, on peut avancer que le modèle rationnel qui fonde les nouveaux documents et catégories comptables informe, en partie, les pratiques non seulement des comptables mais également des directeurs d'entreprises et autres acteurs économiques. En cela, la nouvelle comptabilité peut être abordée comme un des moteurs du changement social en cours aujourd'hui dans la société chinoise.

Nous allons alors tenter d'analyser l'action de ces cadres comptables sur les représentations (à travers l'émergence de nouvelles rubriques) chez les acteurs eux-mêmes, mais aussi sur les pratiques grâce à l'utilisation des bilans comptables. L'étude vise à la fois les décisions bancaires (octroi ou refus de prêts) et les décisions gouvernementales de mises en faillite. À cette fin, nous comparerons les entretiens et informations collectés lors de nos enquêtes de terrain du milieu des années 1990 avec ceux recueillis à la fin des années 1990 et début des années 2000. Bien qu'étant assez proches dans le temps, ces observations permettent d'ores et déjà de percevoir certains changements.

\subsubsection{MILIEU DES ANNÉES 1990 .}

La réforme chinoise introduit de nouvelles catégories comptables (prêt, investissement, subvention, dette et créance). Force est d'admettre que trois ans après leur introduction, elles sont encore largement confondues dans les discours des directeurs d'entreprise et du personnel bancaire : tous considèrent les comptes clients comme des prêts et très peu distinguent une subvention, d'un prêt bancaire ou d'un investissement de l'État ${ }^{14}$. Cette vision globalisante ou moniste est encore prégnante du fait même que ces fonds proviennent d'une seule caisse, celle du Parti. Ainsi l'exprime un directeur d'entreprise : « Nous devons de l'argent à des entreprises d'État qui nous doivent de l'argent, nous devons de l'argent aux banques d'État, nous devons de l'argent à l'État, nous sommes une entreprise d'État. Pour moi, c'est absurde, c'est le Parti communiste qui doit au Parti communiste ».

Hormis ces difficultés d'ordre perceptuel, la mise en place de la réforme entraîne nombre de difficultés et frictions dont l'octroi de prêts bancaires nous offre une illustration assez marquante. Tout accord de financement fait désormais l'objet d'une analyse préalable de bilan. Or, cette pratique entre en conflit avec d'autres encore en vigueur, toutes étant largement imbriquées. Analysons-les brièvement :

- les prêts pré-affectés sont appelés, en chinois, des "prêts conformément aux ordres " ou des "prêts fixés en fonction de politiques ». Voici ce que nous en dit une personne travaillant au service des prêts d'une agence bancaire pékinoise : "particulièrement nombreux, ces prêts sont, en quelque sorte, imposés par une autorité : telle entreprise ne peut plus payer ses dépenses; les échelons supérieurs nous envoient alors un document nous demandant de lui attribuer un prêt tandis qu'on pressent fortement qu'il ne sera jamais remboursé. Parfois, le document descend de notre succursale, parfois du comité du Parti de Pékin, ou encore de l'État. L'octroi de ces prêts, finalement, dépend moins des profits économiques que des points de vue administratif et politique ${ }^{15}$ ";

- la pression du gouvernement ou du comité du Parti à l'échelle locale est tout aussi forte lorsque les difficultés d'une entreprise l'empêchent de payer les salaires. Le souci d'éviter des troubles sociaux entraîne les collectivités à biaiser les normes d'octroi des prêts ; 
- ce même phénomène se retrouve au niveau de la banque dont la survie dépend aussi de la santé de ses clients. Écoutons ce cadre : « ... il y a des entreprises en situation difficile dans lesquelles je n'aime pas descendre. Comme le Groupe X, en pleine crise. Nous devons lui accorder des prêts parce que les relations entre lui et nous sont à la vie à la mort : s'il fait faillite, notre banque en sera très affectée. Nous disposons d'une agence de cent personnes dont l'activité est en grande partie dédiée à ses paiements et ceux de ses usines et entités auxiliaires. Aussi, devons-nous trouver des moyens pour lui accorder des prêts";

- les relations personnelles constituent aussi un autre paramètre qui contrevient aux nouvelles règles de financement. Un expert-comptable : " quand je veux emprunter de l'argent à une banque, j'utilise les relations interpersonnelles (guanxi). Je suis chef comptable d'une entreprise, tu travailles dans une banque, si nos relations sont bonnes, nous sommes des amis; avant nous étions à la même école ou dans la même unité de travail ${ }^{16}$, peu importe ma situation financière, je te donne un peu d'argent ou d'autres avantages et tu n'as pas peur puisque la banque n'est pas à toi. Alors tu attaches peu d'égards aux documents financiers : ta situation financière, tes profits, peu importe, tu as des amis dans la banque, ça suffit."

En somme, l'impact économique de la réforme comptable était, trois ans après sa mise en route, relativement limitée. La nouvelle comptabilité ne semblait pas avoir rencontré dans l'état des structures économiques et sociales chinoises les moyens de se rendre efficace.

\subsubsection{FIN DES ANNÉES 1990-DÉBUT DES ANNÉES 2000}

La dissociation des catégories comptables est, dans les discours, largement plus prononcée durant cette période ; le terme "prêt ", par exemple, n’est plus utilisé pour parler des créances clients mais uniquement des prêts bancaires même si cette dissociation n'est pas toujours très nette dans l'esprit des directeurs d'entreprise ; d'autant plus que certaines réformes récentes, en particulier celles consistant à " transformer des dettes en actions" (zhai gai gu) alimentent la confusion ${ }^{17}$.

Les mêmes remarques peuvent être faites à propos des prêts bancaires. L'évolution générale est à l'octroi de prêts sur la base de critères économiques et donc à la lumière de l'analyse des états financiers de l'entreprise. Cette tendance est fortement et régulièrement réaffirmée par le Premier ministre chinois de l'époque, Zhu Rongji : des sanctions sont prises contre des dirigeants d'agences ou succursales bancaires attachés aux anciennes méthodes. Dans le même temps, des mesures sont prises pour couper le lien entre banques et gouvernement au niveau local. Pour autant, la réitération des discours et la publicité faite autour des sanctions nous invitent à penser que l'évolution ne se produit pas aussi vite que le souhaiteraient les autorités.

En revanche, là où cette réforme est d'une efficacité remarquable, c'est dans la mise en faillite et les plans de licenciement depuis 1997. Le président de l'époque, Jiang Zemin, dans sa déclaration au XVe congrès du Parti en septembre 1997, prend une position tranchée sur la question des sureffectifs des entreprises d'État, et déclare qu'il faut "choisir une réduction du personnel pour une augmentation des résultats". Cette manière de poser le problème, comme les conclusions qui en découlent, est complètement nouvelle en Chine. Une telle perspective s'élève depuis que les salariés n'apparaissent dans les comptes de l'entreprise qu'à travers leurs rémunérations considérées comme des charges ${ }^{18}$. De la même manière, les pertes des entreprises d'État deviennent un problème central 
pour le gouvernement. Or, c'est par la réforme comptable qu'elles sont devenues visibles ${ }^{19}:$ le compte de résultat révèle l'étendue de leurs pertes, le bilan fait apparaître leur haut niveau d'endettement, l'importance de la dette inter-entreprises ainsi que les créances douteuses ou irrécouvrables.

Le chef du gouvernement, Zhu Rongji, annonce alors que le problème des pertes dégagées par les entreprises d'État, doit être réglé dans les trois ans et menace de démissionner dans le cas contraire. Il s'ensuit une pression forte et continue sur les autorités locales. Les entreprises d'État font l'objet d'une grande campagne d'audit avec pour principal objectif de repérer celles qui souffrent de pertes importantes et chroniques. Des plans de licenciement, de préretraites et parfois de liquidation sont alors déployés. Le bilan, quoique difficile à chiffrer précisément, est considérable. Sur l'ensemble de la période 1994-2000 (plus fortement entre 1997 et 2000), on estime qu'auraient été relégués en xiagang (littéralement ceux qui sont descendus de poste, ou, autrement dit, ceux qui n'ont plus de poste) 36 à 55 millions de personnes, soit entre un tiers et la moitié des employés d'entreprises d'État répertoriés en 1994 (Rocca, 2000). Ces estimations ne rendent pas complètement compte de l'ampleur du phénomène ; en particulier, elles laissent de côté la pratique de la mise en retraite anticipée : parfois dès 40 ans pour les femmes, 50 ans pour les hommes, avec un salaire très réduit.

Il est à présent peu discutable que la réforme comptable, du fait de la reconnaissance du profit au cœur du dispositif, encadrée par le pouvoir central, a entraîné de gigantesques conséquences sociales.

\section{Conclusion}

À partir de cette analyse de la réforme comptable chinoise, nous pouvons dégager différents éléments d'ordre général.

D'abord, il nous semble clair que la comptabilité est l'objet d'enjeux sociaux, de négociations et de luttes entre les acteurs intéressés (par exemple entre les ministères industriels chinois et le Bureau national des Statistiques) ; ses règles, catégories et documents sont le résultat du rapport des forces en présence. Tout modèle de normalisation comptable n'est finalement qu'une construction modelée par les facteurs politiques, sociaux et culturels d'un pays (Nobes, Parker 1988)

Ensuite, la comptabilité véhicule des modèles de représentation de la réalité, en particulier de l'entreprise et de ses objectifs. Plus généralement, il faut reconnaître que les règles comptables ne se contentent pas de produire une mesure de l'entité économique : par la symbolique adoptée et les choix sous-jacents, elles contribuent discrètement à façonner la représentation intellectuelle que l'individu se fait de la firme et des rapports qu'elle entretient avec les autres agents.

Enfin, la comptabilité n'est pas seulement un produit de l'histoire mais elle est aussi productrice d'histoire en ce qu'elle participe à la structuration des pratiques managériales et au développement de l'esprit de calcul. Cet aspect apparaît dans un entretien avec un directeur d'entreprise : "Avec cette nouvelle comptabilité, les profits se révèlent clairement, je vois comment les augmenter : il faut compter ; j'ai besoin de tel matériau, à tel endroit il est à 9600 yuan la tonne, à tel autre à 10000 yuan, le premier représente une économie, il faut que nous ayons un esprit de calcul ; de la même manière il faut diminuer les gaspillages en matières premières et en produits finis ».

Ce constat corrobore l'idée que la formation de la comptabilité d'entreprise a accompagné le développement de la rationalité économique et du capitalisme (voir entre autres Weber 1991;

Comptabilité - Contrôle - Audit / Tome 14 - Volume 2 - Décembre 2008 (p. 27 à 42) 
Braudel 1979; Weber 1971 ; Sombard 1932) ${ }^{20}$. Si une réforme comptable peut avoir un impact sur la réalité sociale, c'est là un point toutefois particulièrement difficile à étudier car il faudrait pouvoir isoler "l'effet comptable »; on peut néanmoins souligner que l'effet d'ensemble est possible justement quand l'élément comptable ne reste pas isolé mais trouve des échos, des alliés, des relais et forme corps et système avec eux (d’où la portée limitée de la réforme au milieu des années 1990).

Finalement, il existe un lien de dépendance fort entre système comptable, système de fonctionnement économique et mode de gestion du social. Il est possible de réfléchir à cette relation en prenant appui sur différents travaux d'économie ou de sociologie. Les économistes néo-institutionnalistes ont par exemple montré que la transition d'une économie planifiée à une économie de marché supposait la destruction d'institutions ${ }^{21}$ anciennes et la construction de nouvelles (Vercueil 2001) ; ils ont ainsi mis l'accent sur l'existence de " configurations institutionnelles ", et sur le fait que ce processus est dépendant du chemin déjà parcouru (path dependancy) (North 1990).

Pour notre part, nous considérons l'entreprise d'État chinoise comme une forme sociale particulière (Eyraud 1999) et nous centrons notre analyse sur deux aspects : ses contours et sa qualité essentielle. L'entreprise d'État du temps de l'économie planifiée est d'une part, un ensemble relativement flou qui se prolonge de maintes manières dans l'organisation administrative, politique et sociale ; d'autre part, elle est plus qu'un lieu de travail, plus qu'une communauté de vie, une institution sociale totale au sens d'Erving Goffman $(1968)^{22}$. Le nouveau type d'entreprise que les réformes visent à mettre en place a, en revanche, des contours bien délimités pour devenir de véritables entités, essentiellement économiques. Ces deux types de formes sociales étant objectivés dans des documents comptables particuliers, on peut ainsi « lire » ce changement dans la réforme de la comptabilité. Dès lors, cette dernière nous semble être non seulement une discipline en elle-même mais également une entrée intéressante pour l'analyse de phénomènes et processus plus larges ${ }^{23}$. 


\section{Notes}

1. Lire à ce propos l'analyse longitudinale des éditoriaux de cette revue par S. Lefrancq (2004).

2. Voir en particulier les travaux d'A. Hopwood (fondateur et rédacteur en chef de cette revue depuis sa création en 1976), P. Miller, M. Power, etc. Pour une vision relativement synthétique de ces travaux même si un peu ancienne, on peut se référer à Hopwood et Miller (Eds), 1994.

3. Voir par exemple les travaux de M. Capron, B. Colasse, C. Colette, J. Richard, Y. Lemarchand, M. Mangenot, M. Nikitin.

4. La lecture des différents articles de l'ouvrage dirigé par M. Capron (2005) le démontre également.

5. Là réside peut-être l'intérêt de cette contribution : écrit conjointement par deux enseignantschercheurs, l'un en sciences de gestion, l'autre en sociologie, l'article s'appuie sur six enquêtes se déroulant entre 1993 et 2001, représentant dixhuit mois de terrain :

- un recueil documentaire le plus large possible recouvrant à la fois des manuels de comptabilité d'avant et après la réforme (dont la première étape, la plus marquante, eut lieu en 1993), des articles de quotidiens (cette réforme fut très médiatisée en Chine) et revues comptables, des plans comptables et financiers de secteurs industriels avant et après la réforme, les documents financiers (d'avant et après la réforme) de certaines des entreprises dans lesquelles nous nous sommes rendus;

- la réalisation d'une centaine d'entretiens réalisés en langue chinoise (l'un des deux auteurs, C. Eyraud, maîtrise le mandarin) auprès de quatre types d'acteurs : a) personnes travaillant dans des PME d'État: directeurs, directeurs financiers, directeurs du personnel, secrétaires du Parti, président du syndicat, comptables ; b) personnes travaillant dans des services bancaires en relation avec ces entreprises, en particulier dans les services de prêt et donc utilisant des bilans d'entreprises ; c) officiels en charge des questions comptables au Ministère des Finances; d) chercheurs et enseignants-chercheurs au sein d'instituts de sociologie, économie et comptabilité, qui ont surtout été pour nous des informateurs, des aides à la compréhension des phénomènes. Beaucoup de ces acteurs (en particulier des catégories a et d) ont été interviewés plusieurs années de suite. Les entretiens portaient à la fois sur les pratiques et les représentations liées à la question comptable (on pouvait ainsi demander à un directeur financier de nous aider à lire et comprendre les documents financiers de son entreprise, en particulier ceux de 1992 et ceux de 1993 ; à un directeur d'entreprise ou à un personnel bancaire comment il utilisait les documents financiers dans ses décisions ...) ; - l'observation participante à deux colloques internationaux organisés par le ministère des finances chinois, l'un en 1993 en observation, le second en 1994 en tant qu'interprète (une délégation française était présente) ; le premier était destiné à faire le point sur la réforme comptable de 1993, le second à en étudier les « nécessaires " développements.

Ce travail de terrain puis son analyse ont été réalisés par l'auteur sociologue de cet article. Les résultats, ainsi qu'une partie des données recueillies ont été retravaillés de concert par les deux auteurs.

6. Nos sources, outre les entretiens réalisés, sont essentiellement de langue chinoise ; les informations en langue plus accessible sont rares; on peut se référer à Huang, Mo (2001), Tang (1994) ou Gervais, Wang (1992).

7. D'ailleurs, jusqu'au milieu des années 1980, la comptabilité n'existe pas en tant que discipline indépendante, elle est simplement considérée comme une spécialité des finances publiques.

8. Les autres moyens de paiement, tels les chèques ou les effets de commerce sont considérés comme des « outils capitalistes » et ont été abolis au début des années 1950.

9. Nous avons là aussi travaillé essentiellement sur des sources en langue chinoise, mais une explicitation plus précise de cette réforme est présentée dans Huang and Mo (2001), Tang (1994) ou Nobes, Parker (1988) et en langue française dans Évraert, Ding (2000) ou Morin et al. (1996).

10. Les nouvelles dispositions ne doivent pas nous faire oublier les "lignes de continuité " qui peuvent exister avec la tradition comptable chinoise (cf. Goody 1999, p. 100-105, ainsi que Shanghai 
University 1987, p. 165-190). C'est le système de l'économie planifiée qui est en rupture quasitotale avec la tradition comptable impériale et républicaine. Il est d'ailleurs intéressant de noter qu'en même temps que se créent des groupes d'experts et des commissions autour de la réforme comptable dans les années 1980, se développent les recherches portant sur l'histoire de la comptabilité chinoise " pré-Révolution ".

11. Il devient d'autre part possible pour les entreprises de réaliser des provisions pour créances douteuses ; le principe de prudence est ainsi introduit en comptabilité. Pendant l'économie planifiée, c'est l'État qui était, au bout du compte, responsable de tout achat, vente, perte ou profit, et l'on ne pouvait pas douter de sa solvabilité. L'activité économique devient en Chine une activité à risque. Finalement, la réforme comptable supprime le principe « d'utilisation des crédits conformément à leur affectation"; les directeurs d'entreprise sont ainsi censés acquérir une plus grande liberté quant à l'utilisation de leurs capitaux.

12. Il y aurait beaucoup à dire sur cette question des externalités (Callon 1999; Friedberg, Musselin 1999). Si l'on s'en tient à l'objectivation de ces externalités dans la comptabilité, on peut signaler les travaux d'un groupe de chercheurs composé en particulier de comptables, visant l'élaboration d'un bilan sociétal: "l'entreprise peut enrichir ou au contraire appauvrir son milieu par des coûts externalisés plus ou moins maîtrisés, non seulement en matière d'approvisionnement, de pollution, mais aussi en capital humain. (...) Ce qui est désormais nouveau, c'est le besoin d'évaluer les actions réalisées pour assumer cette responsabilité sociale ou à l'inverse les externalités négatives que fait subir l'entreprise à son environnement. (...) Cela ne peut se faire qu'en changeant les grilles de lecture à l'aune desquelles nous jugeons la bonne santé de l'entreprise, et cela implique d'avoir une autre conception de la performance. (...) L'idée de bilan sociétal est née dans ce contexte avec pour objectif d'identifier les éléments de production et d'échanges de l'entreprise envers la société. »(Capron, Leseul 1997, p. 30).

13. Les catégories collectives de perception et d'action, les formes collectives de classification sont des objets d'étude de la sociologie depuis E. Durkheim. L'analyse de cette activité de catégorisation (faire rentrer le monde social dans des catégories) et de classification sociale a connu un renouveau et un développement au cours des années 1980. On peut par exemple citer les travaux de R. Salais sur l'émergence et l'institutionnalisation des catégories de " chômage " et de "chômeur" (Salais, Baverez, Reynaud, 1986). C. Eyraud (2004) a tenté de montrer l'intérêt pour la science comptable de ce type d'approche.

14. Le responsable d'une agence bancaire française installée en Chine nous signalait que les directeurs d'entreprises chinoises venaient presque toujours le trouver en lui demandant d'investir dans leur entreprise et lui s'évertuait à leur expliquer qu'il n'investissait pas mais prêtait de l'argent.

15. Les grandes banques d'État sont ainsi confrontées à des logiques clairement contradictoires : le gouvernement, au travers des réformes, leur demande d'agir en tant qu'entités économiques responsables de leurs profits et de leurs pertes et donc d'octroyer des prêts sur des critères économiques, et en même temps leur ordonne d'octroyer un prêt à telle ou telle entreprise en difficulté.

16. L'unité de travail ou "danwei " est le nom donné en Chine à toute organisation : un ministère, une entreprise, un hôpital, une école, etc.

17. Les prêts bancaires non remboursés par des entreprises d'État (donc des dettes des entreprises d'État vis-à-vis de banques d'État) sont, sous certaines conditions, transformées en actions que les mêmes banques détiennent sur ces entreprises. Cela permet, assez facilement, de désendetter les entreprises et de diminuer les créances irrécouvrables des banques.

18. G. Lecointre, à propos de ce choix de comptabilisation des salariés, soulignait qu' "il est à craindre que (ce choix) soit celui de comptes non seulement aveugles mais potentiellement mortifères. Ces pratiques comptables pourraient être mises en examen et avoir quelques comptes à rendre au procès des licenciements s'il s'ouvrait un jour. " (dans Colasse 2000, p. 982). 
19. Dans le même sens, P. Miller et T. O’Leary (1987) analysaient le développement des pratiques de coût de revient standard en Grande-Bretagne et aux États-Unis à partir du début des années 1900 comme " making inefficiency visible and calculable» (p. 238).

20. Cela dit, ces auteurs et d'autres qui suivront ne s'entendent ni sur la place ni sur la force de la comptabilité dans ce processus. W. Sombart et M. Weber y accordent une place prépondérante, F. Braudel une place parmi d'autres (la lettre de change aurait eu d'après lui une force supérieure). J. Goody estime également qu'elle est " une composante parmi d'autres dans l'élaboration progressive d'un système complexe de production et d'échange" (1999, p. 104), et réfute, en s'appuyant sur l'histoire du commerce et de la comptabilité au Proche-Orient et en Asie (Chine et Inde), l'idée wéberienne selon laquelle la rationalité occidentale et la comptabilité en partie double seraient intrinsèquement liées au capitalisme, en seraient des conditions de possibilité. Pour J. Goody, les concepts abstraits de profit, de firme, d'évaluation du capital seraient moins indissociablement liés au développement de l'économie industrielle moderne qu'intrinsèques aux

\section{Bibliographie}

Amblard, M. (2000). Les multiples frontières de l'entité comptable. La Revue du financier 123 : 73-85.

Amblard, M. (2002). Modélisation comptable et théorie des conventions. Paris : L'Harmattan.

Amblard, M. (2004). Conventions et comptabilité : vers une approche sociologique du modèle. Comptabilité - Contrôle - Audit numéro spécial (juin) : 47-68.

Boltanski, L. (1982). Les cadres. Paris : Éditions de Minuit.

Braudel, F. (1979). Civilisation matérielle, économie et capitalisme, $15^{e}-18^{e}$ siècle (2). Paris : Colin.

Boyer, R. (1993). La grande transformation à l'Est : une lecture régulationniste. Cahier de recherche, CEPREMAP.

Callon, M. (1999). La sociologie peut-elle enrichir l'analyse économique des externalités ? Essai sur la toutes premières grandes entreprises commerciales en Asie comme en Europe (1999, chap. 2 : "rationalité et ragioneria: la tenue des livres de comptes et le miracle économique ", p. 66-106).

21. Pour une analyse de la notion d'institution chez les économistes, on peut se référer à Vercueil (2001, p. 268-277).

22. "C'est une caractéristique fondamentale des sociétés modernes que l'individu dorme, se distraie et travaille dans des endroits différents, avec des partenaires différents, sous des autorités différentes, sans que cette diversité d'appartenances relève d'un plan d'ensemble. Les institutions totales, au contraire, brisent les frontières qui séparent ordinairement ces trois champs d'activité [...]. Le caractère essentiel des institutions totales est qu'elles appliquent à l'homme un traitement collectif conforme à un système d'organisation bureaucratique qui prend en charge tous ses besoins." (Goffman 1968, p. 47-48).

23. Un numéro de la revue «Comptabilité, Contrôle, Audit» (revue de l'Association francophone de Comptabilité) publié en 2004 et coordonné par Eve Chiapello est consacré à « La sociologie de la comptabilité "; il a permis de nourrir une réflexion que nous prolongeons dans cet article.

notion de cadrage-débordement. In Innovations et performances (Eds, Foray, D., Mairesse J.). Paris : Éditions de l'EHESS, 399-431.

Capron, M. (1985). La Comptabilité. Paris: La Découverte.

Capron, M., Leseul, G. (1997). Pour un bilan sociétal des entreprises. Revue des Études coopératives mutualistes et associatives (RECMA) $266: 28-41$.

Capron, M., Chiapello, E., Colasse, B., Mangenot, M., Richard, J. (2005). Les normes comptables internationales, instruments du capitalisme financier. Paris : La Découverte.

Centre des Jeunes Dirigeants d'entreprise(CJD), (1996). L'entreprise au XXI siècle. Paris : Flammarion.

Colasse, B. (Ed.) (2000). Encyclopédie de comptabilité, contrôle de gestion et audit. Paris : Economica.

Desrosieres, A., Thevenot, L. (1988). Les catégories socio-professionnelles. Paris : La Découverte. 
Ding, Y. (2001). Les facteurs de risque hypothéquant la réussite de la réforme comptable en Chine. Comptabilité - Contrôle - Audit 7 (2) : 25-43.

Ding, Y., Evraert, S. (2002). Les enseignements d'une comparaison de la comptabilité sociale des entreprises en Chine et en France. Comptabilité - Contrôle - Audit 8 (2) : 287-310.

Donzelot, J. (1984). L'invention du social, essai sur le déclin des passions politiques. Paris : Fayard.

Ding, Y., Evraert, S. (2000). Comptabilité en Chine. In Encyclopédie de comptabilité, contrôle de gestion et audit (Ed., Colasse, B.). Paris : Economica, 291-300.

Eyraud, C. (2004). Comptabilité (publique et d'entreprise) et sociologie, ou l'analyse sociologique des catégorisations sociales. Comptabilité - Contrôle - Audit, numéro spécial (juin) : 29-45.

Eyraud, C. (1999). L'entreprise d'État chinoise: de "l'institution sociale totale "vers l'entité économique ? Paris : L'Harmattan.

Ferrandon, B. (2002). Les leçons de l'affaire Enron, Les Cahiers Français 309: 69.

Friedberg, E., Musselin, C. (1999). Les externalités d'un point de vue sociologique. Quelques remarques. In Innovations et performances (Eds, Foray, D., Mairesse J.). Paris : Éditions de l'EHESS, 447-451.

Gervais, M., Wang, H.T. (1992). Les principes de la comptabilité chinoise. Revue française de Comptabilité 240 : 89-103.

Goffman, E. (1968). Asiles. Paris : Éditions de Minuit. Goody, J. (1999). L'Orient en Occident. Paris : Seuil.

Hopwood, A., Miller, P. (Eds.) (1994). Accounting as social and institutional practice. Cambridge: Cambridge University Press.

Hopwood, A. (2000). Editorial : Celebrating and reviewing. Accounting, Organizations and Society $8(25): 3-4$.

Huang, A., Mo R. (2001). Accounting in China in transition : 1949-2000. Australia : Griffith University.

Laville, J.L. (1997). Le renouveau de la sociologie économique. Cahiers internationaux de Sociologie $103: 229-235$.

Lefrancq, S. (2004). Recherche et action : la comptabilité dans son contexte. Une étude de la politique éditoriale d'Accounting, Organizations and Society. Comptabilité - Contrôle - Audit, numéro spécial (juin) : 297-315.

Mangenot, M. (1976). La comptabilité au service du capital. Paris : J-P. Delarge éditeur.

Miller, P., O’leary, T. (1987). Accounting and the Construction of the Governable Person. Accounting, Organizations and Society 3 (12): 235-265.

Miller, P. (2000). How and Why Sociology Forgot Accounting. Conférence pour le $25^{\mathrm{e}}$ anniversaire d'Accounting, Organizations and Society, Londres.

Morin, A., Pairault, T., Wu, C. (1996). Le plan comptable des entreprises industrielles chinoises. Paris: EHESS et Ordre des experts-comptables.

Nobes, C.W., Parker, R.H. (1988). Issues in Multinational Accounting. Oxford : Philip Allan.

North, D.C. (1990). Institutions, institutional change and economic performance. Cambridge: Cambridge University Press.

Rocca, J.L. (2000). La montée du chômage dans la Chine urbaine. China Perspective 59 : 38-51.

Salais, R., Baverez, N., Reynaud, B. (1986). L'invention du chômage. Paris : PUF.

Shanghai University of Finance and Economics, University of Texas at Dallas (1987). Accounting and Auditing in the People's Republic of China. Shanghai : Shanghai University Press.

Sombard, W. (1932). L'apogée du capitalisme. Paris : Payot.

Tang, Y. (ed.) (1994). Accounting and Finance in China, a Review of Current Practice. Hong Kong : Longman Group.

Thevenot, L. (1985). Les investissements de forme. Cahiers du Centre d'études de l'emploi 29: 21-71.

Vercueil, J. (2001). Transition et ouverture de l'économie russe (1992-2002) - Pour une économie institutionnelle du changement. Paris : L'Harmattan.

Weber, M. (1971). Économie et sociétés. Paris : Plon.

Weber, M. (1991). Histoire économique - Esquisse d'une histoire universelle de l'économie et de la société. Paris : Gallimard. 